\title{
OlloBot - Towards A Text-Based Arabic Health Conversational Agent: Evaluation and Results
}

\author{
Ahmed Fadhil \\ Department of Computer Science \\ Universita Degli Studi di Trento \\ Trento, Italy \\ ahmed.fadhileunitn.it
}

\author{
Ahmed AbuRa'ed \\ Information \& Communication Technologies \\ Universitat Pompeu Fabra \\ Barcelona, Spain \\ ahmed. aburaed@upf .edu
}

\begin{abstract}
We introduce OlloBot, an Arabic conversational agent that assists physicians and supports patients with the care process. It doesn't replace the physicians, instead provides health tracking and support and assists physicians with the care delivery through a conversation medium. The current model comprises healthy diet, physical activity, mental health, in addition to food logging. Not only OlloBot tracks user daily food, it also offers useful tips for healthier living. We will discuss the design, development and testing of OlloBot, and highlight the findings and limitations arose from the testing.
\end{abstract}

\section{Introduction}

According to World Health Organisation (WHO), poor diet and physical inactivity have tremendous implications on individuals health (Michie et al., 2009; Hard et al., 2012). Healthy diet helps protect against malnutrition in all its forms, as well as noncommunicable diseases (NCDs) (Michie et al., 2009). Middle eastern countries are among the mostly affected nations by poor diet and physical inactivity and its effect on cardiovascular diseases (Organization et al., 2010, 2012). According to WHO report, total deaths caused by NCDs are among the highest in countries such as, Saudi Arabia, Iraq, Qatar, and Bahrain (See Table-1). This is an evident for a global risk and there is a need for nationwide approach to help mitigate or prevent this escalation.

By 2025, AI systems could be involved in everything from population health management, to virtual assistants capable of answering specific patient queries. Overall, AI has the potential to improve outcomes by 30 to 40 percent while cutting

\begin{tabular}{l|c}
\hline \multicolumn{2}{c}{ The NCDs and Middle East (WHO) } \\
\hline \multicolumn{1}{c}{ Countries } & $\%$ of total deaths by NCDs \\
\hline Saudi Arabia & Cardiovascular diseases $=46 \%$ \\
\hline Qatar & Cardiovascular diseases $=24 \%$ \\
\hline Iraq & Cardiovascular diseases $=33 \%$ \\
\hline Bahrain & Cardiovascular diseases $=26 \%$ \\
\hline
\end{tabular}

Table 1: Middle East Countries and NCD Burden.

treatment costs by as much as 50 percent (Koh et al., 2011). Conversational agents, an example of AI powered systems can communicate with users through an intelligent conversation using a natural language, they can aid doctors in enhancing productivity and enabling them to respond to patients quickly. For example, doctors could interact with a diet chatbot to recommend the appropriate food to their patients. Patients could also engage with this chatbot to get instant information about their dietary choices.

Currently, some ways to use healthcare chatbots include: scheduling doctor appointments based on the severity of the symptoms, monitoring the health status and notifying a human nurse immediately if the parameters are out of control, helping home-care assistants stay informed about patients evolution. However, current health conversational agents are mature for English language but still in their infancy for specific demographics and languages. For example, Hebrew and Arabic are far more complex languages for conversational agents. To ensure timely health information delivery to Arabic users, the patient should be able to tell the bot what symptoms they are experiencing and receive medical advice. However, this is not possible with current approaches, since they merely focus on generic approaches. Hence, in this research we highlight the role of chatbots to provide health services to individuals with lan- 
guage barriers. The approach targets demographics who are not bilingual and can speak only Arabic language. This is important, since from Table1 we see the burden of NCDs in Arabic speaking countries. We propose OlloBot, an Arabic conversational agent able to converse with users and handle daily tasks about diet, physical activity, mental wellness and coping. The bot can also track users daily food and keep a record of their daily dietary habits. To our knowledge, few studies exists on conversational agents that supports Arabic language, able to flexibly converse and handle dialogues. However, no study exists that have considered the health benefit achievable with language specific conversational agents. Few platforms provide support to Arabic language technology to build interactive and intelligent conversational agents. This might be due to the complexity of the language and the resource scarcity available to support it. Our approach relies on IBM Watson Conversation API (IBM bluemix) ${ }^{1}$ to handle the dialogue structure and Telegram Bot Platform to build the chatbot. We tested OlloBot with 43 Arabic speaking users and presented the findings in this paper.

\section{Background Research}

Although cognitive behavioral therapeutic (CBT) apps have demonstrated efficacy, still they are characterized by low adherence rate (Fitzpatrick et al., 2017). Conversational agents, on the other hand, may offer a convenient and engaging alternative of giving support at any time. A work by Graf et al., (Graf et al., 2015) built a chatbot that assimilates into daily routines. The bot communicates with user and gathers nutrition data. Keeping interaction with the bot final is a good design practice, however building a great dialogue flow is also essential to ensure smooth user interaction. This includes the way the bot handles several user interactions and requests. Zaghouani et al., (Zaghouani et al., 2015) presented a correction annotation guidelines to create a manually corrected nonnative (L2) Arabic corpus. The work extends a large scale Arabic corpus and its manual corrections to include manually corrected non-native Arabic learner essays. The approach uses annotated corpus to develop components for automatic detection and correction of language error to help standard Arabic learners and improve the quality

\footnotetext{
${ }^{1}$ https://www.ibm.com/cloud-computing/bluemix/it
}

of Arabic text produced. Chatbots could be used as language learning tools, to access information, to visualise the context of a corpus and to give answers to specific questions (Abu Shawar, 2005). A work by Shawar et al., (Abu Shawar, 2005) built a general language chatbot that supported different language among which is Arabic and English. The study also used different corpora structure, such as dialogue, monologue and structured text to build the dialogue model.

A work by Ali et al., (Ali and Habash, 2016) presented BOTTA, an Arabic dialect chatbot that communicates with users using the Egyptian Arabic dialect. Another work by Shawar et al., (Shawar and Atwell, 2009) described a technique to access Arabic information using chatbot with natural language processing (NLP) tools. The bot provides responses to capture the logical ontology of a given domain using a set of pattern-template matching rules. The literature shows no studies considering conversational agent as assistive tool for Arabic speakers. Which could provide instant access to health information and data, and assist physicians in providing a follow up to the patients. Conversational agents are great in handling repetitive tasks which consumes most of the healthcare providers time. Sundermeyer et al., (Sundermeyer et al., 2014) build a two translation recurrent neural models. The first one is a word-based approach using word alignments, while the second presents phrase-based translation models that are more consistent with phrase-based decoding. The models are capable of improving strong baselines in BOLT task for Arabic to English.

\section{OlloBot Architecture}

To provide the required level of Natural Language Understanding (NLU), we built the Arabic dialogue states on IBM Watson Conversation ${ }^{2}$, which defines the conversation flow and dialogue states. The platform provides Artificial Intelligence support to catch different user intents and entities. The OlloBot starts with the user sending a message to the bot (OlloBot) running on Telegram Bot Platform ${ }^{3}$. The application provides the user with the topics they can chat about, then based on user selection the bot forwards the request to IBM Watson Conversation cloud. The conversation slots takes user input and provides them with

\footnotetext{
${ }^{2}$ https://www.ibm.com/watson/services/conversation/

${ }^{3}$ https://telegram.org/blog/bot-revolution
} 
relevant answer after checking their intent, entity and condition of the conversion. The logical parts of the dialogue are handled by a Node.js wrapper to handle unmatched dialogues by the dialogue flow ( Figure-1 illustrates the high-level architectural view of OlloBot).

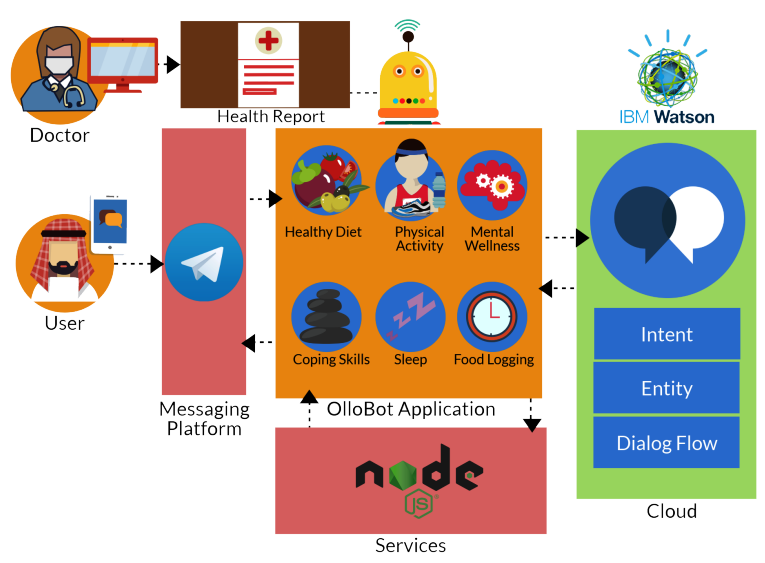

Figure 1: OlloBot High-level Architecture.

The bot detects patterns in user phrasing and correlates them with the intents defined. For example, conversing about users daily meal and asking users to enter their breakfast, they can either provide a list of food items or send an emojis as a list. The bot can extract the intend from each item or emoji and build a list of food items. In principle, this induces the system to recognize food items inserted by users. The need for conversational agents rises due to the on-demand 24/7 access to care. However, with millions of people globally who struggle with poor diet, poor exercise, anxiety and depression, there isn't enough healthcare provider or psychologists to provide the necessary care. Moreover, in some parts, there is limited technological services supporting native languages and sometimes services are practically nonexistent. Due to the associated cost for care, providing conversational agents as a novel technology that is cost effective, efficient and targets the right demographics is essential. OlloBot bridges these gaps and provides Arabic users with a quick and accessible health services. The bot converses about diet, exercise, emotion and provide coping skills. It can also ask the user to $\log$ their daily food and gather data about their preferred style of diet and exercise day, exercise, sleep. The fact that OlloBot is supported by Watson and easily accessible by many users makes it able to support many users at the same time.
The chatbot gathers users interaction data, and performance indicators from their interaction. These data are saved in a database in the form of accessible reports by the caregivers. OlloBot acts as a health assistants, meaning that it offers help and support rather than treatment.

\subsection{User Intents}

The intents refers to what the users want from the bot or what's the objective behind a user's input. For instance, the user intents hi, hello, hi OlloBot are translated to the intent "Greetings", whereas the intents food logging, daily food, logging data...etc are all translated into "Food Logging". We carefully sketched the dialogue scope the bot covers and those that it does not (see Table2 for a list of user intents). The dialog flow structure was designed in collaboration with a healthcare clinic. After defining the flows for key in-

\begin{tabular}{c|c}
\hline \multicolumn{2}{c}{ User Intents } \\
\hline Greetings & Coping Skills \\
\hline Diet and nutrition & Daily meals Logging \\
\hline Mood & Jokes \\
\hline Physical activity & Conditions \\
\hline Anything else & Farewells \\
\hline
\end{tabular}

Table 2: The User Intents

tents, we defined the bot responses or follow up, once the task is performed. When the user starts a conversation, the bot can either track user dialogue or leave it at resolution and reset. The bot can switch between intends, based on user input. Since the interaction medium is conversational, users can switch intents on the chatbot. For example, while the bot waits for the user to provide relevant information about their physical activity, the user can ask the bot to insert their breakfast. OlloBot switches between the topics given in the above table, by detecting user intent from the conversation. To accurately handle this switching, we designed additional flows represented by conditions. Although this adds flexibility to the interaction, however, it can also create additional cognitive load.

The next bot response depends on what the user will say or choose from the buttons. For example, after the user provides their daily food logging, the bot can move on and ask them about their suitable time to be notified again. Users can come back to the chatbot at a later time, with no recollection of 
the task they were trying to accomplish, therefore tracking the dialogue flow is essential to offer a more flexible conversation to the users. Finally, to handle fallbacks where the bot has no clue about the respond, we designed the "anything else" intent, to handle unhandled intents.

\subsection{Health Report}

The chatbot application provides a health report generated by users interaction with the chatbot. This report contains user activity data, their overall interaction with the application and indications about their overall health. The health measure is mainly about their diet, exercise and stress pattern. All these data are structurally generated by the chatbot and saved into the database. These data can then be accessed by the caregiver to obtain relevant user specific data.

\subsection{Entities}

Entities are the pieces of valuable information hidden in users input. They're important keywords extracted from a sentence. For example, in the utterance "I want to talk about physical activity", the word "physical activity" is detected as an entity, and hence the bot switches to the physical activity topic. Entities focus on defining the topic the user is talking about. This is important to provide the right respond to user questions. We defined a big range of entities to build our dialogue model. These entities represented the four main topics and the daily food logging. In Table-3 we provide the entities listed to structure the dialogue flow.

\begin{tabular}{c|c}
\hline \multicolumn{2}{c}{ Topic Entities } \\
\hline Topics & Meal times \\
\hline Daily times & Weekly times \\
\hline Quantity & Food \\
\hline Numbers & Sport \\
\hline
\end{tabular}

Table 3: The Topic Entities

The above table lists the "Topics" entity, namely diet, physical activity, mood, and coping that the bot converses about. The topics entity also covers users daily food logging. Other entities include the "Meal times" which defines the meal periods per day that includes: breakfast, lunch, snacks and dinner. The "Daily times" refers to the periods of the day, namely morning, noon, afternoon, evening and night. Whereas, "Weekly times" entity includes the period of the day, namely yes- terday, today and tomorrow. The "Quantity" entity refers to the quantity measurements the user might mention in the conversation (i.e., $\mathrm{kg}$, g, tea spoon, bread loaf), whereas the "Numbers" entity refers to the countable number the user might mention. We have also defined a comprehensive list of common Arabic food items and included them in the "Food" entity. Finally, we defined a list of any kind of sports in the "Sport" entity. The entity list was accompanied by a list of synonym to add flexibility for the entity detection. This is important to detect user intents and sentiments from their conversation. While there has been a lot of research on sentiment analysis in English, the amount of research and datasets for Arabic language is still limited. A work by Alayba et al., (Alayba et al., 2017) built a sentiment analysis dataset in Arabic from Twitter data. The study applied machine learning algorithms (i.e., Naive Bayes, Support Vector Machine and Logistic Regression) for the analysis. Another work by Ismail et al., (Ismail and Ahmad, 2004) proposed a new type of recurrent neural network architecture for speech recognition and Backpropagation Through Time (BPTT) learning algorithm to observe differences in alphabet "alif" until "ya".

\subsection{Dialogue Engine}

The dialogue structure was designed for each of healthy diet, physical activity, and mental wellness topics by referencing the Cognitive-behavioural therapy (CBT) (Rothbaum et al., 2000). We based the chatbot dialogue on techniques suggested by the CBT.

The dialogue was then developed on IBM Watson Conversation, where we listed each of the intents and entities and built the dialogue structure and flow. The tasks were intentionally built to be simple to interact with, so to decrease the time and amount of physical and mental efforts needed, and increase user's ability. For example, when asked to log their food, users can either write the list or send an emoji of the food item. Moreover, button replies were provided to further simplify the data logging. Simplifying food tracking process will increase users ability and decrease the learning curve associated to health tracking. This is because interaction with the bot shouldn't be only conversational, since some interactions are better with Graphical UI and others with Conversational UI. 


\subsubsection{Healthy Diet}

Around 2 billion people are overweight, but many are ready to change (Van Itallie, 1985). However, according to studies (Mann et al., 2007) temporary fixes to old habits makes people to regain their weight. OlloBot acts as an interactive AI-powered diet tracking bot that converses with individuals in a friendly way directly through the Telegram messaging application. The goal is to give instant advices with each meal eaten and help improve the eating habits on the go. Once the conversation is executed, OlloBot starts conversing about user's diet and asks them questions about their eating habits and highlights the values associated with healthy diet. For example, OlloBot stresses the fact that following a diet rich in vegetables and fruits helps decrease escalation into overweight, obesity or even chronic conditions, and the detrimental effects associated otherwise.

\subsubsection{Physical Activity}

Studies (Cooney et al., 2014; Mead et al., 2009; Artal et al., 1998; Byrne and Byrne, 1993) have shown that exercise can treat mild to moderate depression as effectively as antidepressant medication and with no associated side-effects. Our conversational agent chats with users about their physical activity and daily energy level, and can provide personalized plans and keep track of workout progress by storing relevant user inputs. Whether the user wants to stay fit, loose weight, or get toned, OlloBot can later provide an efficient and consistent workout plan while keeping track of the progress. Although the bot is not considered as a tool to loose weight through exercise, but rather a supportive tool to help individuals track their exercise and improve in on the go, making users conscious about their health habits also makes them more likely to set aside time for physical activities in the future.

\subsubsection{Mental Wellness}

Mental health refers to our overall psychological well-being. This includes the way we feel about ourselves, the quality of our relationships and the ability to manage our feeling. OlloBot chats with users about mental health and asks them questions about their stress, sleep and other measures relevant to their mental wellbeing. There exists several mental health chatbots that provide support at different stages of mental illness. For exam- ple, $\mathrm{X} 2 \mathrm{AI}^{4}$ created a set of chatbots for mental health applications. Their flagship AI, Tess, helps patients in tandem with their doctors by providing resources on cognitive-behavioral therapy, medication side effects, and questionnaire automation. Woebot ${ }^{5}$ is a mood tracking chatbot with personality. Backed by scientific research, Woebot can help reduce depression, share CBT resources, and learn from conversations over time. Finally, Joy ${ }^{6}$ uses a chatbot approach to mental health. She offers options for both individuals and therapists.

\subsubsection{Coping Skills}

Being mentally or emotionally healthy is more than being free of depression, anxiety, or other psychological issues. Rather than the absence of mental illness, mental health refers to the presence of positive characteristics. With OlloBot, we handle this by providing coping skills and mindfulness support through clever motivational quotes, relevant workout suggestions mixed with some health facts to help users understand the benefit of health and wellness. The bot provides the uses with tips about coping with stress, diet, exercise, sleep, and mindfulness. The quotes and suggestions are all based on best recommendations provided by the World Health Organisation (WHO) (Michie et al., 2009). Coping skills are important, since finding a moment to take a few deep breaths and quiet your mind is a great way to relieve stress and improve your overall health.

\subsubsection{Daily Food Logging}

While many food-logging tools are already on the market, most are either too complicated or boring for the average individual. OlloBot keeps a food diary, tracks calories, and provides basic nutritional tips based on user's eating habits. We wanted to make the food logging process simpler, more engaging, and more informative for the users. The bot tracks user meals, by asking them to insert each meal items. This helps to know more about users diet. With the NLP capabilities offered by IBM Watson Conversation, the bot supports any food combinations. The user can even $\log$ their food by sending emojis of the food items. Once the food logging is done, the bot asks the user about their preferred time to recheck with them. OlloBot is in its early stages, due to the time

\footnotetext{
${ }^{4}$ https://x2.ai/

${ }^{5}$ https://woebot.io/

${ }^{6}$ http://www.hellojoy.ai/
} 


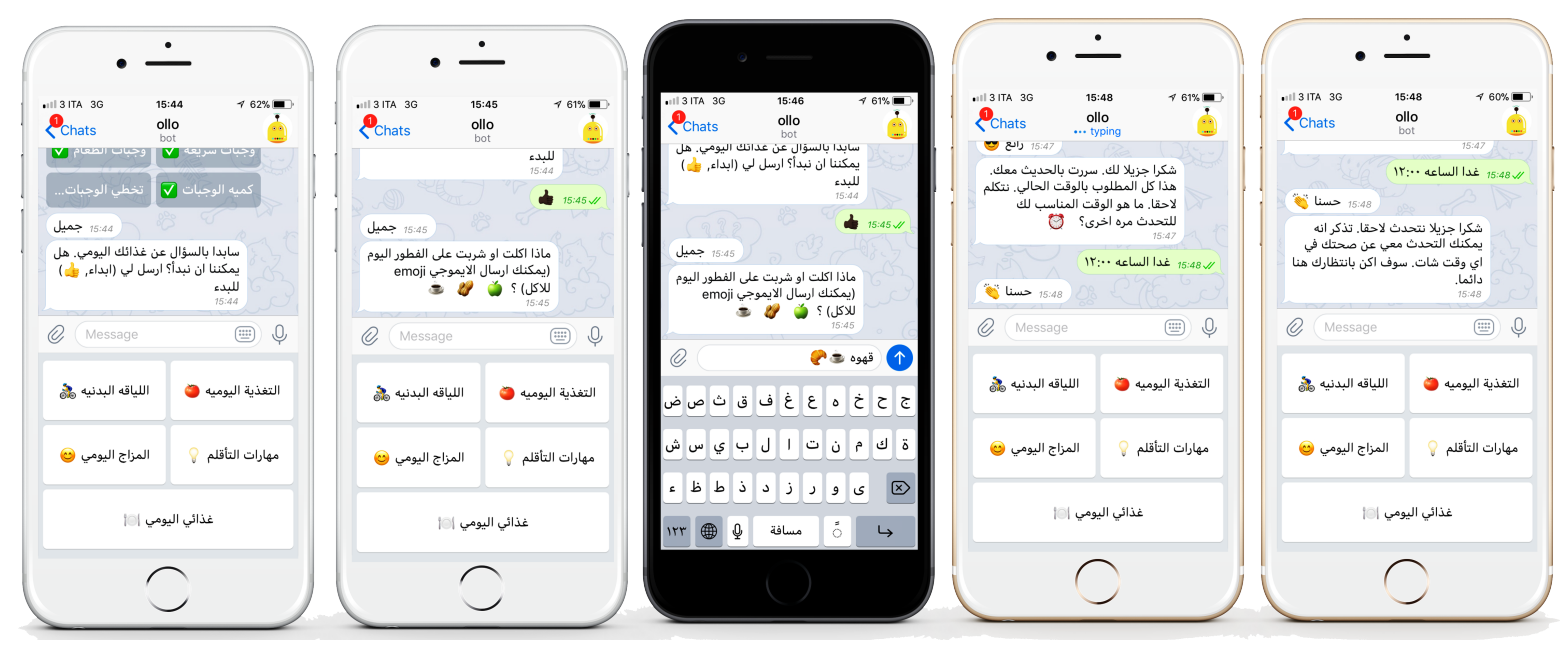

Figure 2: Daily Food Logging Conversation with OlloBot.

required to build and integrate new features, then validate it through testing with real users. We understand that existing food logging apps are now far ahead of us in terms of calorie tracking, precision, and various interface features. However, we aim to close this gap and simplify the food tracking/logging and feedback providing process in the future (see Figure-2 for the daily food logging with OlloBot).

\section{OlloBot Platform}

The dialogue flow in OlloBot were handled with IBM Watson Conversation, whereas the logical part of the dialogue were handled with a Node.js implementation. Our choice of IBM Watson was due to its language support, to our knowledge it was the only conversational agent dialogue building platform that supports Arabic. We deployed the bot on Telegram Bot Platform and built the components to handle user requests and responses. We used various UI elements made available by Telegram and integrated them into our dialogue model.

\section{OlloBot - USE Questionnaire}

After building and integrating OlloBot with Telegram Bot Platform, we conducted a user experiment with 43 Arabic speaking users. The dialogue and various questions the bot asks were all based on a WHO questionnaire we extracted about health and wellbeing (Kessler and Üstün, 2004; Parslow and Jorm, 2000; , WHO et al.(2017; Organization et al., 2006). After testing the chatbot, we performed a survey analysis to test various as- pects of the bot. The questionnaire is based on a standard framework, namely USE Questionnaire (Lund, 2001) to measure the usability of the chatbot. The framework tests four items of usability within a product. It consists of 30 questions to test the usefulness, easy of use, ease of learning and satisfaction with the application. The questionnaires are organised in a scale of $1-5$, where 1 is "strongly disagree" and 5 is "strongly agree". We describe the experiment settings and results in the following sections.

\subsection{Participants Demographics}

The user demographics consisted of both male $(n=26)$ and female $(n=17)$ participants with an age range of $20-65$ years old for both gender. We checked the participants familiarity with tracking devices (e.g., any diet, sleep, physical activity, or mood tracking application) and chatbot applications (see Table-4 for the results). There was no significant differences in both cases with either tracking devices (e.g., wearable, sensor, or mobile applications) or chatbot applications. The majority of participants have shown no familiarity with tracking devices $(n=26)$ and chatbot applications $(n=27)$. We provided additional questions at the end of the survey to evaluate their overall experience with the chatbot. A reimbursement of $5 €$ was given to all participants in the form of Amazon coupon.

\subsection{Experiment}

We distributed the chatbot to all the Arabic speaking participants recruited from Iraq. After carrying out the experiment for 1 week, we collected data 


\begin{tabular}{l|c|c}
\hline \multirow{2}{*}{ Gender } & Female & 17 \\
\cline { 2 - 3 } & Male & 26 \\
\hline \multirow{2}{*}{ Age } & Mean & 29.8 \\
\cline { 2 - 3 } & Std. Dev & 9.28 \\
\hline \multirow{2}{*}{ Tracking devices familiar } & No & 26 \\
\cline { 2 - 3 } & Yes & 17 \\
\hline \multirow{2}{*}{ Chatbot familiar } & No & 27 \\
\cline { 2 - 3 } & Yes & 16 \\
\hline
\end{tabular}

Table 4: Participants Demographics.

through questionnaire to test the four main points relevant to the usability of OlloBot.

\subsubsection{Usefulness}

This item helps to test how useful the participants perceived the application. The usefulness includes measuring whether the bot helps the user to be more effective and productive and enhance their control over their daily life activities. For example, "I believe is effective to track my health" evaluates whether the user thinks the bot is effective in tracking their health.

\begin{tabular}{|c|c|c|c|c|}
\hline Dimensions & Mean & $\begin{array}{l}\text { Std. } \\
\text { Dev. }\end{array}$ & $\begin{array}{l}\text { t value } \\
(\mathrm{df}=42)\end{array}$ & p value \\
\hline Usefulness & 381 & 0.373 & 6.695 & $p<.01$ \\
\hline ase of Us & 626 & 3944 & 10.405 & $p<.01$ \\
\hline & 3.942 & 0.5341 & 11.564 & for learnability compared \\
\hline Satisfaction & 3.505 & 0.3667 & 9.031 & former group $(\mathrm{t}(41)=-2.46, \mathrm{p}<.01)$. \\
\hline
\end{tabular}

Table 5: The Results from the USE Questionnaire.

\subsubsection{Ease of Use}

This point helps understand the ease of use aspect. It measures easiness, simplicity and user friendliness of the application (chatbot). This point also measures the steps and effort required to achieve the goal set and whether its easy to recover from mistakes. For example, to measure the effort required for each step, we asked users to provide their scale for "It requires the fewest steps possible to accomplish what I want to do with it". We performed a descriptive statistics about the four items checked in the overall experience (see Table-6). Figure-3 below lists each of the overall bot reliability (Figure-3a), and the participants overall experience (Figure-3b, Figure-3c and Figure-3d).

\subsubsection{Ease of Learning}

This point measures the learnability of the tool. We measure whether its quick to learn and easy to remember each time. For example, the question "I quickly became skilful with it" checks for how quickly a skill is learned using the chatbot.

\subsubsection{Satisfaction}

This step checks for user's overall satisfaction with the chatbot. It checked whether the users are satisfied and would recommend the tool to others and how entertaining they perceived it. For example, the question "I would recommend it to a friend or family member" checks whether the user would recommend the bot to their relatives or friends.

We performed descriptive statistics on the results and reported them in Table-5. One Sample t test showed that averages for all four scales were statistically significantly different from the middle value (value $=3$, see Table-5). Further analyses were performed considering gender, familiarity with tracking technology, and familiarity with chatbot as between factors. No differences among the four usability dimensions were observed between male and female participants, and no differences emerged between participants who frequently use or not use tracking devices. A significant difference for the "ease of learning" scale was comparing participants who were articipants who were not. The latter group rethe former group $(\mathrm{t}(41)=-2.46, \mathrm{p}<.01)$.

\subsubsection{Overall Experience}

This part evaluated the overall experience with OlloBot and is not part of the USE questionnaire. We tested user satisfaction with the reliability of OlloBot, their overall experience with the bot from different perspectives (see Table-7 for the experience comparison). We have checked whether users like chatbots or rather use a mobile application. Finally, we considered the list of most positive and negative aspects the users mentioned during the survey. In addition, we asked the users about the features they would like to see/use in feature versions of OlloBot.

\section{Discussion}

This work was the first to evaluate the application of NLP powered health tools into language and context scarce domains. The work involved initial design phase, which involved researchers and health experts in the context of healthy lifestyle 

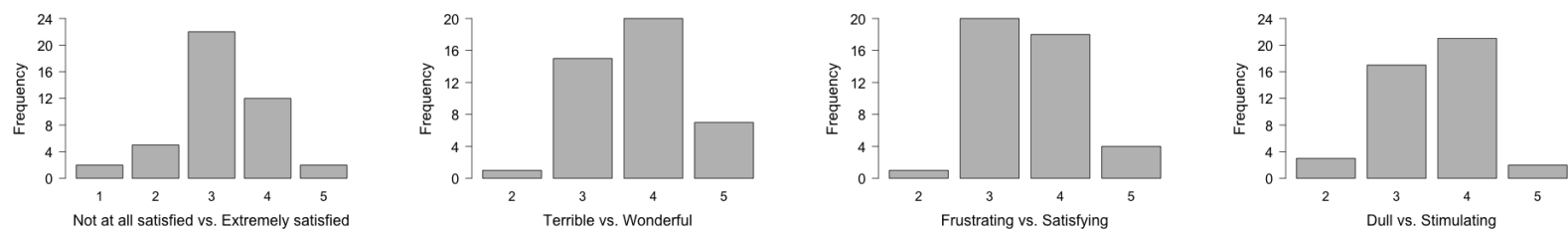

(a) Overall Reliability Satisfac-(b) Participants Overall experi-(c) Participants Overall experi-(d) Participants Overall experition. ence.

Figure 3: The Overall Experience with OlloBot.

\begin{tabular}{c|c|c|c|c|c}
\hline & App vs. Chatbot & Not at all satisfied vs. Extremely satisfied & Terrible vs. Wonderful & Frustrating vs. Satisfying & Dull vs. Stimulating \\
\hline Valid & 43 & 43 & 43 & 43 & 43 \\
\hline Mean & 3.860 & 3.163 & 3.767 & 3.581 & 0.6980 \\
\hline Std. Dev. & 0.9900 & 0.8710 & 0.7508 & 2.000 & 0.70028 \\
\hline Min & 1.000 & 1.000 & 2.000 & 2.000 & 5.000 \\
\hline Max & 5.000 & 5.000 & 5.000 & 5.000 \\
\hline
\end{tabular}

Table 6: The Descriptive Statistics for Chatbot Experience.

\begin{tabular}{|c|c|c|}
\hline \multicolumn{3}{|l|}{ Overall Experience } \\
\hline Questions & Scale $=1$ & Scale $=5$ \\
\hline How satisfied are you with the reliability of this chatbot? & Not at all satisfied & Extremely satisfied \\
\hline Can you rate your experience with the chatbot: & Terrible & Wonderful \\
\hline Can you rate your experience with the chatbot: & Frustrating & Satisfying \\
\hline Can you rate your experience with the chatbot: & Dull & Stimulating \\
\hline $\begin{array}{l}\text { Would you rather use the chatbot or prefer a mobile app to } \\
\text { track your everyday health? }\end{array}$ & Use an app & Use a chatbot \\
\hline
\end{tabular}

Table 7: Users Overall Experience With OlloBot.

promotion. Involving the health expert was necessary to guarantee the real context applicability of the application. The dialog structure was built in collaboration with a dietitian who described the steps necessary when building a dialog with a patient. We then applied this design paradigm into our dialog engine. The existing work has several limitations worth mentioning. Since most of the research work was carried out in Italy in collaboration with Spain, the dietitian involved in designing the dialog was a native Italian. Yet, the guidance obtained from the expert was translated into Arabic language and integrated into the Watson dialog engine. We also acknowledge that the size of the participants and the period of experiments, although provide good indications, are not enough to conclude any long-term effect. Future work will consider building a standalone dialog model and including a larger user samples and over an extended period.

\section{Conclusion}

Chatbots can be a trustworthy assistant, like a caring nurse, which provides registration services and patient follow up. Medical practices can rely on chatbots to capture leads and provide 24/7 support to existing patients, answering their simple, repetitive questions using a pre-designed answers. It will not offer a diagnosis, but it can remind patients to take their drugs or help them check for an unusual side effect. Most users showed interest towards social intelligence of the bot. Hence, tone and empathy matters, people can be turned off if the experience is too robot-like or casual, so we should opted for a polite tone. In summary, chatbots offer a great user experience to patients by just chatting with the bot to get relevant answers to their queries. Future work will integrate OlloBot into a health coaching system and make it specific to support Arabic speaking, diabetic patients. 


\section{References}

Bayan Aref Abu Shawar. 2005. A corpus based approach to generalise a chatbot system. Ph.D. thesis, University of Leeds.

Abdulaziz M Alayba, Vasile Palade, Matthew England, and Rahat Iqbal. 2017. Arabic language sentiment analysis on health services. arXiv preprint arXiv:1702.03197.

Dana Abu Ali and Nizar Habash. 2016. Botta: An arabic dialect chatbot. In COLING (Demos). pages 208-212.

Michal Artal, Carl Sherman, and Nicholas A DiNubile. 1998. Exercise against depression. The Physician and sportsmedicine 26(10):55-70.

A Byrne and DG Byrne. 1993. The effect of exercise on depression, anxiety and other mood states: a review. Journal of psychosomatic research 37(6):565-574.

Gary Cooney, Kerry Dwan, and Gillian Mead. 2014. Exercise for depression. Jama 311(23):2432-2433.

Kathleen Kara Fitzpatrick, Alison Darcy, and Molly Vierhile. 2017. Delivering cognitive behavior therapy to young adults with symptoms of depression and anxiety using a fully automated conversational agent (woebot): A randomized controlled trial. JMIR Mental Health 4(2):e19.

Bettina Graf, Maike Krüger, Felix Müller, Alexander Ruhland, and Andrea Zech. 2015. Nombot: simplify food tracking. In Proceedings of the 14th International Conference on Mobile and Ubiquitous Multimedia. ACM, pages 360-363.

Eat Smart Play Hard, Healthy Kansas Schools, In-Class Physical Activity Breaks, and Blast Off Game. 2012. Physical activity. Fitness and health: Internatio .

Saliza Ismail and A Ahmad. 2004. Recurrent neural network with back propagation through time algorithm for arabic recognition. Proceedings of the 18th ESM Magdeburg, Germany pages 13-16.

Ronald C Kessler and T Bedirhan Üstün. 2004. The world mental health (wmh) survey initiative version of the world health organization (who) composite international diagnostic interview (cidi). International journal of methods in psychiatric research 13(2):93121.

Hian Chye Koh, Gerald Tan, et al. 2011. Data mining applications in healthcare. Journal of healthcare information management 19(2):65.

Arnold M Lund. 2001. Measuring usability with the use questionnaire12. Usability interface 8(2):3-6.

Traci Mann, A Janet Tomiyama, Erika Westling, AnnMarie Lew, Barbra Samuels, and Jason Chatman. 2007. Medicare's search for effective obesity treatments: diets are not the answer. American Psychologist 62(3):220.
Gillian E Mead, Wendy Morley, Paul Campbell, Carolyn A Greig, Marion McMurdo, Debbie A Lawlor, et al. 2009. Exercise for depression. Cochrane Database Syst Rev 3.

Susan Michie, Charles Abraham, Craig Whittington, John McAteer, and Sunjai Gupta. 2009. Effective techniques in healthy eating and physical activity interventions: a meta-regression. Health Psychology 28(6):690.

World Health Organization et al. 2006. Global strategy on diet, physical activity and health: a framework to monitor and evaluate implementation .

World Health Organization et al. 2010. WORLD HEALH REPORT (The): Health Systems Financing: the path to universal Coverage (Arabic). World Health Organization.

World Health Organization et al. 2012. World health day 2012: ageing and health: toolkit for event organizers .

Ruth A Parslow and Anthony F Jorm. 2000. Who uses mental health services in australia? an analysis of data from the national survey of mental health and wellbeing. Australian and New Zealand Journal of Psychiatry 34(6):997-1008.

Barbara Olasov Rothbaum, Elizabeth A Meadows, Patricia Resick, and David W Foy. 2000. Cognitivebehavioral therapy. .

B Abu Shawar and Eric Atwell. 2009. Arabic questionanswering via instance based learning from an faq corpus. In Proceedings of the CL 2009 International Conference on Corpus Linguistics. UCREL. volume 386.

Martin Sundermeyer, Tamer Alkhouli, Joern Wuebker, and Hermann Ney. 2014. Translation modeling with bidirectional recurrent neural networks. In EMNLP. pages 14-25.

Theodore B Van Itallie. 1985. Health implications of overweight and obesity in the united states. Annals of internal medicine 103(6_Part_2):983-988.

World Health Organization (WHO et al. 2017. Obesity and overweight factsheet from the who. Health .

Wajdi Zaghouani, Nizar Habash, Houda Bouamor, Alla Rozovskaya, Behrang Mohit, Abeer Heider, and Kemal Oflazer. 2015. Correction annotation for nonnative arabic texts: Guidelines and corpus. In LAW@NAACL-HLT. pages 129-139. 